\title{
FEVER IN SYSTEMIC LUPUS ERYTHEMATOSUS
}

anna beatriz gomes souza duarte (Hospital federal Servidores do Estado, Rio de Janeiro, RJ, Brasil), Elisa Guimarães Motta (Hospital federal Servidores do Estado, rio de janeiro, RJ, Brasil), Adib Chicre Mansur (Hospital federal Servidores do Estado, rio de janeiro, RJ, Brasil), guilherme salles de escobar Gonçaves (Hospital federal dos Servidores do Estado, rio de janeiro, RJ, Brasil), Larissa Barros de Oliveira (Hospital federal dos Servidores do estado, rio de janeiro, RJ, Brasil), gabriellen vitiello (Hospitala federal Servidores do Estado, rio de janeiro, RJ, Brasil)

\section{BACKGROUND}

Patients can be challenging, Systemic lupus erythematosus (SLE) can lead to end stage kidney disease and recent data demonstrated that patients that receive kidney transplants have decreased mortality both from cardiovascular disease and from infections.

\section{CASE REPORT}

A 31-year-old caucasian female was referred to our unit for investigation of a fever of $38-38.9^{\circ} \mathrm{C}$, lasting for 30 days. At admission, she presented hair loss, oral ulcers and a malar rash. Blood tests revealed anemia, low complement and leucopenia. Chest and abdomen CT scans showed laminar right pleural effusion, multiple lymph nodes and adipose densification of the adjacent tissue to the kidney allograft. Her previous medical history included a SLE and antiphospholipid syndrome diagnosis in 2009, that evolved to end-stage renal failure. She received a live-related renal transplant in 2015 and was maintained on an immunosuppressive regimen with mycophenolate anda tacrolimus. She also hads a previous history of skeletal tuberculosis that complicated with irreversible paraplegia in 2017. In 2018, she was admitted to the hospital to treat an urinary sepsis. At that same year she also had loss of her renal and returned to hemodialysis. In this scenario, our main diagnostic hypotheses were: SLE disease activity, infections (disseminated Tuberculosis, cryptococcosis, histoplasmosis, paracoccioidomycosis), common in our environment, lymphoproliproliferative disease, or renal graft chronic rejection. Treatment for disease activity and broad spectrum antibiotics were started but the fever persisted. All serologies were negative. Among the possibilities, hyposthesis of rejection to the renal transplantation was proposed. Then She had been submitted to resection of allograft. Patient responded very well and almost immediately recovered from the fever. Histopathologic examination confirmed the renal rejection.

\section{CONCLUSION}

The differential diagnosis of fever in SLE cases requires careful investigation. In this case, it was important to carefully evaluate the possible causes of fever in transplanted patients with SLE, as well as the prognosis and outcomes of the renal graft it self. 